preferred study budget and leave process explained at induction. 47 of 48 respnders sought access to their study budget, 46 of 48 responders sought use of budget for external course and exam fees fudning, 27 of the 48 responders struggled to get study leave and 27 of 48 responders felt the current leave process was not satisfactory with 14 opining that there was scope for improvemement. Conclusion. The responses were collated by the trainee rep as a PowerPoint presentation containing graphical representation of trainees' views regarding their study leaves and budget access. The survey results were made aware to the local board at the subsequent RAP Oversight Committee meeting to seek response and new guidance. There is a new system in place for study leave and study budgets, overseen by Health Education England. Overall, the survery attempted to understand and collate trainees' wants and needs, in effect improving trainee experiences.

\section{Developing a virtual mock casc for trainees}

Laura Somerville $^{1 \star}$, Peter McMurray ${ }^{2}$, Vivian Sing ${ }^{3}$, Stephanie Campbell ${ }^{4}$ and Meta McGee ${ }^{5}$

${ }^{1}$ South Eastern HSCT, Northern Ireland Medical and Dental Training Agency; ${ }^{2}$ Western HSCT, Northern Ireland Medical and Dental Training Agency; ${ }^{3}$ Southern HSCT, Northern Ireland Medical and Dental Training Agency; ${ }^{4}$ Northern HSCT, Northern Ireland Medical and Dental Training Agency and ${ }^{5}$ University of Hull, Northern Ireland Medical and Dental Training Agency

${ }^{*}$ Corresponding author.

doi: 10.1192/bjo.2021.440

Aims. The restrictions experienced due to the COVID-19 pandemic had impacts on how clinical teaching and assessment is conducted. The Royal College of Psychiatrists decided to run the final part of the membership exam, the Clinical Assessment of Skills and Competencies (CASC) online for the first time in September 2020. We aimed to prepare candidates in the Northern Ireland deanery for this by developing a virtual mock examination using the Zoom platform.

Method. In previous years, higher psychiatry trainees in the Northern Ireland deanery have run successful face to face mock examinations to help pre-membership trainees prepare for the CASC. We adapted some of this material to our virtual examination. 16 stations were run in total, in two circuits of eight. These stations were mapped to the Royal College CASC blueprint. Higher trainees were recruited to act as examiners, with core trainees acting as simulated patients. The mock examination was advertised through the local deanery and all candidates sitting in September availed of the opportunity (a total of 8 trainees).

Zoom was used as the platform due to ease of use, familiarity and breakout room function. Each station formed one breakout room, and a facilitator moved candidates between rooms and provided timing prompts. Instructions were emailed to candidates in advance.

A comfort break was provided between circuits. At the end of the mock examination, everyone was returned to the main room and examiners gave general feedback and tips. Individual feedback was provided by collating mark schemes for each candidate, which included free text feedback, and sending these via email.

Result. Despite the evident challenges involved, the mock CASC ran smoothly. There was one minor delay of approximately $3 \mathrm{~min}$ utes due to technical difficulties, which was easily recouped. We obtained qualitative feedback from candidates which was positive, with trainees commenting that they felt "more at ease ... less worried" about a digital exam, and that it was "efficient and effective".

All candidates who sat the mock examination were successful in the face to face CASC sitting which followed in September.
Conclusion. We were able to successfully adapt what was previously an in-person mock CASC exam to the new digital format in a way that reflected how the actual CASC exam will run, and it was considered beneficial preparation by the candidates who sat this mock. This has improved trainee experience at a time when many teaching opportunities have been suspended.

\section{The use of drama and theatre in enhancing communication skills of psychiatry trainees: a pilot study}

Laura Stevenson $^{1 \star}$, Farooq Khan ${ }^{2}$, Qusai Bharmal ${ }^{3}$, Opeyemi Odejimi ${ }^{2}$, Sheliza Samnani ${ }^{2}$ and Polly Wright ${ }^{4}$

${ }^{1}$ North Staffordshire Combined Healthcare NHS Trust; ${ }^{2}$ Birmingham and Solihull Mental Health NHS Foundation Trust; ${ }^{3}$ Black Country Healthcare NHS Foundation Trust and ${ }^{4}$ The Hearth Centre ${ }^{\star}$ Corresponding author.

doi: 10.1192/bjo.2021.441

Aims. Various methods have been employed in the development of communication skills. This pilot study was designed to assess the acceptability, feasibility and cost-effectiveness of a specially designed workshop exploring the use of drama and theatre in enhancing the self-reported communication skills of psychiatry trainees. As a secondary aim, it assessed if the value of the improvements translated both into clinical practice and to training situations, including success in the Royal College of Psychiatrists (RCPsych) Clinical Assessment of Skills and Competencies (CASC) examination.

Method. A three-day drama and theatre workshop was organised in the West Midlands Deanery in conjunction with specialist instructors from performing arts at the Hearth Centre, Birmingham. The Tension State technique developed by Jacques Lecoq and Forum Theatre approach, were some of the methods employed to enable participants to develop the softer, but essential communication skills required for effective practice. Work was also undertaken focussing on self-regulation. Fourteen trainees completed the first day of the workshop. This pilot study utilised a mixed methodology to evaluate participants' views of the perceived impact of using drama and theatre to enhance their communication skills. Feedback was obtained from organisers and facilitators specifically relating to feasibility and cost effectiveness. Data were collected from participants using pre and postworkshop questionnaires and focus groups.

Result. All participants reported a positive and enjoyable experience, indicating that the approach was acceptable to those involved. The facilitators deemed this more novel approach to enhancing communication skills feasible, and cost effective and concluded that there was scope to incorporate it into routine psychiatry training in the area. It was however identified that the content of the workshop could be condensed, reducing the length therefore to two days. There was a notable increase in participants' self-reported confidence in their communication skills post compared to pre-workshop. Trainees reported utilising the techniques in day-to-day practice. All of those participants who undertook the CASC examination during the workshop were successful, although it would be too presumptive to assume a causative effect. The workshop was completed without any adverse events and there were no concerns from a safety perspective.

Conclusion. Drama and theatre, as a novel approach, appears to have noticeable benefits in enhancing the communication skills of psychiatry trainees. The success of this pilot study in demonstrating acceptability, feasibility and cost effectiveness, suggests that drama 